\title{
Statement of Work for Analytical Services Provided By Pacific Northwest National Laboratory Analytical Chemistry Laboratory
}

Prepared for the U.S. Department of Energy 


\section{LEGAL DISCLAIMER}

This report was prepared as an account of work sponsored by an agency of the United States Government. Neither the United States Government nor any agency thereof, nor any of theit employees, nor any of their contractors, subcontractors or their employees, makes any warranty, express or implied, or assumes any legal liability or responsibility for the accuracy, completeness, or any third party's use or the results of such use of any information, apparatus, product, or process disclosed, or tepresents that its use would not infringe privately owned rights. Reference herein to any specific commercial product, process, or service by trade name, trademark, manufacturer, or otherwise, does not necessarily constitute or imply its endorsement, recommendation, or favoring by the United States Government or any agency thereof or its contractors or subcontractors. The views and opinions of authors expressed herein do not necessarily state of reflect those of the United States Government or any agency thereof.

This report has been reproduced from the best available copy. Avallable in paper copy and microfiche.

Available to the U.S. Department of Energy

and its contractors from

Office of Scientific and Technical information

P.O. Box 62

Oak Ridge, TN 37837

(615) 576-8401

Available to the public from the U.S. Department of Commerce National Technical Information Service

5285 Port Roysl Road

Springfield, VA 22161

(703) $487-4650$

Printed in the United States of Americs

DISCLM-1.CHP (1-91) 


\section{Statement of Work for Analytical Services Provided By Pacific Northwest National Laboratory Analytical Chemistry Laboratory}

Date Published

June 1997

Prepared for the U.S. Department of Energy

Project Hanford Management Contractor for the

U.S. Department of Energy under Contract DE-ACO6-96RL13200 


\section{RELEASE AUTHORIZATION}

Document Number:

HNF-EP-0857-2

This document, reviewed in accordance with DOE Order 1430.1D, "Scientific and Technical Information Management," and DOE G 1430.1D-1, "Guide to the Management of Scientific and Technical Information," does not contain classified or sensitive unclassified information and is:

\section{APPROVED FOR PUBLIC RELEASE}

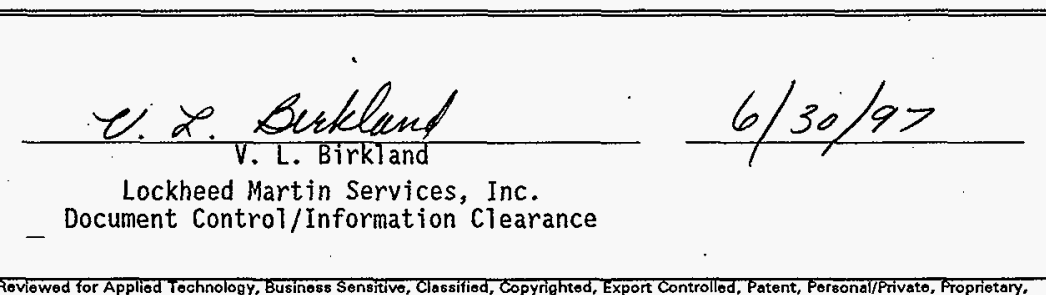

Protoctod CRADA, Trademark, Unclassified Controllod Nuclear Information.

LEGAL. DISCLAIMER. This roport was propared as an account of work sponsored by an agency of the United Statos Govemment. Neither the United States Govemment nor any agency thareof, not any of thoir employeos, ner any of their contractors, subcontractors or their omployoes, makos any warranty, oxpress or implied, or assumes any logal liability or responsibility for the occuracy, completeness, ór any third party's use or the results of such use of any information, apporatus, product, or process disciosed, or represents that its use would not infringe privately owned rights. Reference herein to any specific commercial product, process, or senvice by trade neme, tredemark, manufacturer, or otherwise, does not necessarily constitute or imply its endorsement, recommendation, or favoring by the United States Government or any agency thereof or its contractors or subcontractors. The viows and opinions of authors expressed herein do not necessarily state or reflect those of the United States Govemment or any ageney thereof. Thie report has been reproduced from the best available copy. Printed in the United States of America. Availablo to the U.S. Department of Enorgy and its contractors from the U.S. Department of Energy Office of Scientific and Technical Information, P.O. Box 62, Oak Ridge, TN 37831; Tolephono: 423/576-3401.

Available to the public from the U.S. Department of Commerce National Technical information Service, 5285 Port Royal Road, Springfiold, VA 22161; Telephone: 703/487-4650. 


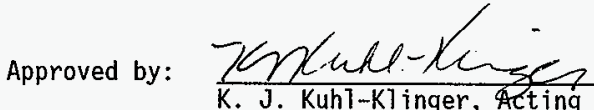
K. J. Kuhl-K] inger, Acting Manager Pacific Northwest National Laboratory Analytical Chemistry Laboratory

Approved by:

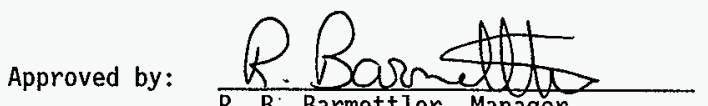

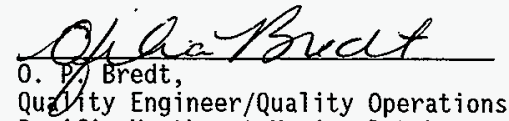

Pacific Northwest NationaT Laboratory Analytical Chemistry Laboratory R. B: Barmettlor, Manager Rust Federa? Services of Hanford Inc. 300 Area LEF

Approved by:

T. A. Dillhoff, Team Leader

B\&W Hanford Company

FFTF Regulatory Comp 7 iance

and ECO, FFTF/MASF

Approved by:

Approved by:

M. S. Wright, Manzger

B\&il Hanford Company

324 Facility Stabilization Project

D. E. Rasmussen, Manager

B\&W Hanford Company

327 Facility

Approved by:
E. J. Bitten, Manager

B\&W Hanford Company

Advanced Fuel Facilities Transition
Efrocter $\frac{6 / 26 / 97}{\text { Date }}$

$\frac{60 / 26 / 97}{\text { Date }}$

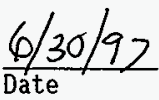

$\frac{6 / 30 / 8 z}{\text { Date }}$

$\frac{6 / 27 / 97}{\text { Date }}$

Approved by: 
HNF-EP-0857-2

Document Title: STATEMENT OF WORK FOR ANALYTICAL SERVICES PROVIDED BY PNNL'S ANALYTICAL CHEMISTRY LABORATORY

Prepared by: $\frac{1-1}{3-12}$

3. R. Perry, Scientist

Rust Federal Services of Hanford Inc.

$\frac{6-30-97}{\text { Date }}$

Air and Water Services

Approved by:

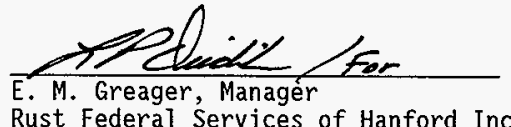

$\frac{<-25-9\rangle}{\text { Date }}$

Approved by:

Air and Water Services

$\frac{6-25-97}{\text { Date }}$

L. P. Diediker, Team Leader

Rust Federal Services of Hanford Inc.

Air and Water Services

Approved by:

J. $\frac{\mathrm{Lubs}}{\mathrm{L} \text { Luke, Team Leader }}$
Rust Federal Services of Hanford Inc

$\frac{b-2 b-97}{\text { Date }}$

Approved by:

Air and Water Services

Approved by:

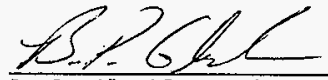

B. P. Gleckler, Advanced Engineer

Rust Federal Services of Hanford Inc.

Air and Water Services

$\frac{6 / 26 / 97}{\text { Date }}$

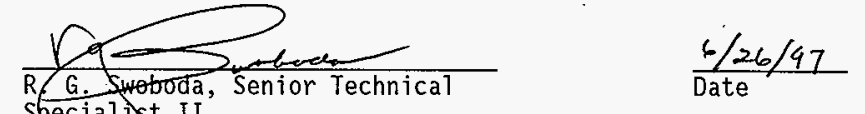

Specialist II

Pacifie Northwest Nationa? Laboratory

Analytical Chemistry Laboratory

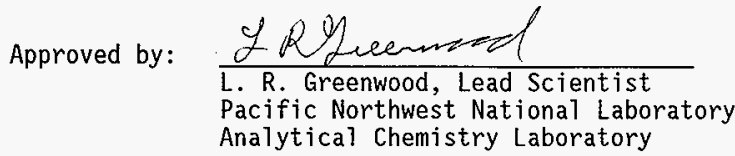

$\frac{6 / 26 / 97}{\text { Date }}$ 
HNF-EP-0857-2

\section{CONTENTS}

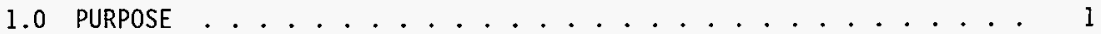

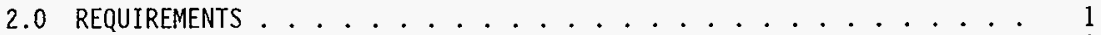

2.1 REGULATORY REQUIREMENTS ................... 1

2.2 CONTRACTED ANALYTICAL LABORATORY . . . . . . . . . . I I

2.3 VARIANCES TO THE STATEMENT OF WORK ............ I I

3.0 REGULATORY REPORTING REQUIREMENTS .............. . 1

3.1 RADIONUCLIDE AIR EMISSIONS REPORT . . . . . . . . . . . . . 2

3.2 ANNUAL ENVIRONMENTAL RELEASES REPORT $\ldots . . . . . . . . . . .2$

3.3 HANFORD SITE ENVIRONMENTAL REPORT . . . . . . . . . . . 2.

3.4 EFFLUENT INFORMATION SYSTEM/ONSITE DISCHARGE INFORMATION

4.0 ANALYTICAL LABORATORY SERVICES AND DATA QUALITY OBJECTIVES . . . . 2

5.0 GLOSSARY ......................... 4

6.0 REFERENCES ......................... 5

\section{TABLES}

1. Sample and Analysis Criteria for the 309 Facility ......... T-1

2. Sample and Analysis Criteria for the 324 Facility . . . . . . . . . . T-2

3. Sample and Analysis Criteria for the 327 Facility . . . . . . . . T-3

4. Sample and Analysis Criteria for the 340 Facility......... T-4

5. Sample and Analysis Criteria for the Fast Flux Test Facility . . . . T-5

6. Sample and Analysis Criteria for the 437 Maintenance and

Storage Facility ................ . . . . . . . . .

7. Environmental Release Summary Reporting Parameters : . . . . . T-7 
HNF-EP-0857-2

This page intentionally left blank. 


\subsection{PURPOSE}

The purpose of this statement of work (SOW) is to establish laboratory analytical criteria and requirements for radioactive airborne emissions measurement sample and/or analysis activities.

\subsection{REQUIREMENTS}

\subsection{REGULATORY REQUIREMENTS}

In accordance with Clean Air Act requirements [ 40 Code of Federal Regulations (CFR) 61.93 and Washington Administrative Code (WAC) 246-247], owners and operators of radioactive airborne emissions units are required to implement a quality assurance program for radioactive airborne emissions measurement activities. In accordance with these requirements, a Quality Assurance Project Plan (WHC-EP-0528-2) has been prepared that governs the quality assurance activities associated with data collection and reporting of radioactive airborne emissions measurements. Included in WHC-EP-0528-2 are requirements for the following:

- Preparation of a SOW for the radioactive airborne emissions measurement program

- Identification of analytical criteria and detection limits for laboratory radioanalytical services.

This document, prepared by Rust Federal Services of Hanford Inc. (RFSH), Air and Water Services (AWS), fulfills these stated requirements for the Project Hanford Management Contract (PHMC)-managed facilities in the 300 and 400 Areas.

\subsection{CONTRACTED ANALYTICAL LABORATORY}

Analytical services for the radioactive airborne emissions measurement program for the PHMC-managed facilities in the 300 and 400 Areas are to be provided by Pacific Northwest National Laboratory's (PNNL) Analytical Chemistry Laboratory.

\subsection{VARIANCES TO THE STATEMENT OF WORK}

Variances to this SOW must be documented by PNNL and approved or acknowledged by the PHMC AWS organization.

\subsection{REGULATORY REPORTING REQUIREMENTS}

The sample data, resulting from the radioactive airborne emissions measurement program for the PHMC-managed facilities in the 300 and 400 Areas, 
HNF-EP-0857-2

support a number of regulatory reporting requirements. A description of the required reports that this program supports is provided in the following sections.

\subsection{RADIONUCLIDE AIR EMISSIONS REPORT}

This report documents Hanford Site radioactive airborne emissions measurements results. Within this report, emissions measurements are used to estimate the annual effective dose equivalent (EDE) received by the hypothetical offsite highest receptor. This report complies with the reporting requirements of WAC 246-247, "Radiation Protection-Air Emissions", and the 40 CFR 61, "Protection of the Environment", "National Emissions Standards for Hazardous Air Pollutants", Subpart H, "National Emission Standards for Emissions of Radionuclides Other Than Radon From Department of Energy Facilities".

\subsection{ANNUAL ENVIRONMENTAL RELEASES REPORT}

This annual report fulfills effluent discharge reporting requirements of U.S. Department of Energy (DOE) Order 5400.1 and DOE Order 5484.1. This report summarizes the degree to which emissions of regulated substances (i.e., radioactive airborne emissions) from Hanford Site facilities comply with applicable regulatory limits.

\subsection{HANFORD SITE ENVIRONMENTAL REPORT}

This report is compiled and published annually by PNNL for U.S. Department of Energy, Richland Operations Office to comply with the requirements of DOE Order 5400.1 .

\subsection{EFFLUENT INFORMATION SYSTEM/ONSITE DISCHARGE INFORMATION SYSTEM}

This report is transmitted electronically to Idaho National Engineering Laboratory (INEL) in Idaho Falls, Idaho for inclusion in the DOE effluent information system/onsite discharge information System (EIS/ODIS) database. This report complies with the requirements of DOE Order 5484.1 and DOE Order 5400.1.

\subsection{ANALYTICAL LABORATORY SERVICES AND DATA QUALITY OBJECTIVES}

PNNL's Analytical Chemistry Laboratory shall provide the following anaiytical and data handling services.

- Analytical services shall meet the analytical requirements listed in Tables 1 through 6. 
- PNNL shall implement a quality assurance $(Q A)$ program that meets the applicable requirements of 40 CFR 61, Appendix B, Method 114, Section 4.0 .

- Quality control test results and laboratory intercomparison scores shall be provided to AWS upon request; the tests shall include accuracy, precision, and background.

- PNNL shall participate in the DOE Inter-Laboratory Quality Assurance Program coordinated by the DOE Environmental Measurements Laboratory, New York, New York, as applicable for requested analyses.

- Upon request, AWS sha11 be permitted to review and approve the QA program.

- Particulate samples for the 340 Tank Vent Stack (340-NT-EX), 324 Facility stack (ESP-324-01-S), 327 Facility stacks (ESP-327-01-S and ESP-327-02-D) sha11 be saved and composited for quarterly specific radionuclide analyses. The composited samples shall be analyzed for plutonium-239/240, plutonium-238, americium-241, and cesium-137 in accordance with 40 CFR 61, Appendix B, Method 114 (refer to tables).

In some cases, sample radioactivity levels might be insufficient to allow for the detection of specific radionuclides. Therefore, as a cost savings measure, gross alpha/beta measurements shall be performed on composite samples before performing specific analyses (not applicable for gamma spectroscopy analyses). If insufficient radioactivity levels are present to allow for possible detection of the specified radionuclides, specific radionuclide analyses shall not be not performed, unless otherwise directed by AwS. Conversely, specific radionuclide analyses shall be performed when gross alpha/beta radioactivity levels are sufficient to allow for possible detection of specific radionuclides.

- PNNL analytical data shall meet a minimum completeness criteria of 90 percent (90\% of samples analyses for each stack shall meet the minimum requirements established in this document).

- Analytical results shall be expressed in the units shown for the respective minimum detectable concentrations (MDC) in Tables 1 through 6 .

- PNNL shall transmit sample analysis results to AWS in an electronic format that can be downloaded into the environmental release summary (ERS) database. Table 7 lists parameters for reporting data via the ERS database.

- All raw data and analytical results shall be retained as QA documents for a minimum of 5 years, as specified by 40 CFR 61 , Subpart $H$ and WAC 246-247 requirements. Upon request, AWS sha 11 be provided with access to the raw data and analytical results. 
- In the event that alpha/beta activity is detected at MDC levels or greater (refer to Tables 1 through 6), PNNL sha11 contact AWS for guidance on performing follow-up analyses.

\subsection{GLOSSARY}

$\begin{array}{ll}\text { AWS } & \text { Air and Water Services } \\ \text { CFR } & \text { Code of Federal Regulations } \\ \text { DOE } & \text { U.S. Department of Energy } \\ \text { DOE-RL } & \text { U.S. Department of Energy, Richland Operations office } \\ \text { EDE } & \text { effective dose equivalent } \\ \text { EDP } & \text { electronic data processing } \\ \text { EIS/ODIS } & \text { effluent information system/onsite discharge } \\ \text { EPA } & \text { U.S. Environmental Protection Agency } \\ \text { ERS } & \text { environmental release summary } \\ \text { FFTF } & \text { Fast Flux Test Facility } \\ \text { HNF } & \text { Hanford Nuclear Facility (document identifier) } \\ \text { INEL } & \text { Idaho National Engineering Laboratory } \\ \text { MASF } & \text { 437 Maintenance and Storage Facility } \\ \text { MDC } & \text { minimum detectable concentrations } \\ \text { PHMC } & \text { Project Hanford Management Contract } \\ \text { PNNL } & \text { Pacific Northwest National Laboratory } \\ \text { QA } & \text { quality assurance } \\ \text { RFSH } & \text { Rust Federal Services of Hanford Inc. } \\ \text { SOW } & \text { statement of work } \\ \text { TT } & \begin{array}{l}\text { turnaround time (elapsed time, in days, from when a } \\ \text { sample is received by the laboratory until the } \\ \text { analysis is reported to AwS) } \\ \text { microcuries per cubic centimeter }\end{array} \\ & \end{array}$


HNF-EP-0857-2

\subsection{REFERENCES}

DOE Order 5400.1, General Environmenta7 Protection Program.

DOE Order 5484.1, Environmental Protection, Safety and Health Protection Information Reporting Requirements.

DOE/RL-96-68, Hanford analytical Services Quality Assurance Requirements Documents, "Volume 4: Laboratory Technical Requirements", U.S. Department of Energy, Richland Operations Office.

WHC-EP-0528-2, Effluent Monitoring Quality Assurance Project Plan for Radioactive Airborne Emissions Data, Westinghouse Hanford Company, Richland, Washington. 
HNF-EP-0857-2

This page intentionaliy left blank. 
Table 1. Sample and Analysis Criteria for the 309 Facility.

\begin{tabular}{|c|c|c|c|c|c|c|c|}
\hline \multicolumn{8}{|c|}{309 Facility, 309-PRTR Stack } \\
\hline \multicolumn{8}{|c|}{ SAMPLE AND ANALYSIS CRITERIA } \\
\hline Sample size & $\begin{array}{l}\text { Type of } \\
\text { analysis }\end{array}$ & Preciston ${ }^{\mathrm{a}}$ & Accuracy & $\begin{array}{c}\text { Samples/year } \\
\text { (frequency) }\end{array}$ & $\begin{array}{l}M O C^{c}, \\
\mu \mathrm{CH} / \mathrm{CC}\end{array}$ & $\begin{array}{l}\text { Thd } \\
\text { days }\end{array}$ & $\begin{array}{c}\text { Unit } \\
\text { price, } \$\end{array}$ \\
\hline $\begin{array}{l}\text { nominal } \\
2 \text { cfm for } \\
\text { period of } \\
\text { collection, } \\
\text { unless } \\
\text { otherwise } \\
\text { indicated }\end{array}$ & $\begin{array}{c}\text { gross } \alpha \\
\text { and } \\
\text { gross } B\end{array}$ & $<20 \%$ & Footnote $b$ & $\begin{array}{c}6 \\
\text { (6 per year) }\end{array}$ & $\begin{array}{c}2.0 \mathrm{E}-15 \\
\text { and } \\
1.9 \mathrm{E}-14\end{array}$ & 14 & 100 \\
\hline
\end{tabular}

a DOE/RL-96-68, Vo] 4 , Table $7-1$, states that duplicate analyses should have $<20 \%$ relative percent difference when the result is $>5$ times the minimum detectable activity.

b Performance evaluation sample/analysis results should meet the minimum acceptability scores specified by the required evaluation studies. Evaluation study requirements are specified in Section 4.0 .

c MDCs shall be as low as reasonably attainable, but shall not exceed the values specified in the table.

d Turnaround times are expressed as calendar days.

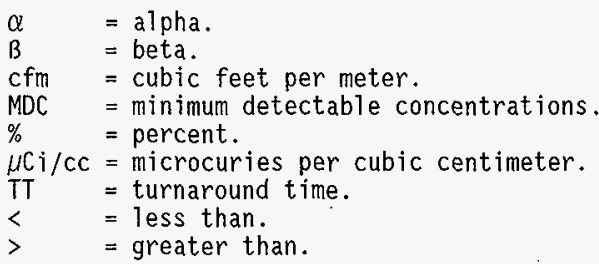


Table 2. Sample and Analysis Criteria for the 324 Facility: (sheet 1 of 2 )

\begin{tabular}{|c|c|c|c|c|c|c|c|}
\hline \multicolumn{8}{|c|}{324 FACILITY, ESP-324-01-S Stack } \\
\hline \multicolumn{8}{|c|}{ SAMPLE AND ANALYSIS CRITERIA } \\
\hline Sample size & $\begin{array}{l}\text { Type of } \\
\text { analysis }\end{array}$ & Precision & Accuracy & $\begin{array}{l}\text { Samples per } \\
\text { year } \\
\text { frequency) }\end{array}$ & MBC & $\begin{array}{r}\mathrm{TT}^{\mathrm{f}} \\
\text { (days) } \\
\end{array}$ & $\begin{array}{l}\text { Unit } \\
\text { prices } \\
\$\end{array}$ \\
\hline \multirow{7}{*}{$\begin{array}{l}\text { nominal } 2.0 \mathrm{cfm} \text { for } \\
\text { period of collection, } \\
\text { unless otherwise } \\
\text { indicated }\end{array}$} & $\begin{array}{c}\text { gross } \alpha \\
\text { and } \\
\text { gross } B\end{array}$ & \multirow[t]{7}{*}{$<20 \%$} & \multirow[t]{7}{*}{ Footnote b } & $\begin{array}{c}26 \\
(1 / 2 \text { weeks })\end{array}$ & $\begin{array}{l}2.0 \mathrm{E}-15 \\
\text { and } \\
1.9 \mathrm{E}-14 \\
\end{array}$ & 14 & 100 \\
\hline & ${ }^{238} \mathrm{Pu}^{\mathrm{d}}$ & & & $\begin{array}{c}4 \\
\text { (1/quarter) }\end{array}$ & $2.1 \mathrm{E}-15$ & Footnote e & 250 \\
\hline & ${ }^{241} \mathrm{Am}^{\mathrm{d}}$ & & & $\begin{array}{c}4 \\
\text { (1/quarter) }\end{array}$ & $1.9 \mathrm{E}-15$ & Footnote e & 250 \\
\hline & ${ }^{3} \mathrm{H}$ & & & $\begin{array}{c}24 \\
\text { (2/month) }\end{array}$ & $1.5 \mathrm{E}-9$ & 45 & 750 \\
\hline & ${ }^{90} S r^{d}$ & & & $\begin{array}{c}4 \\
\text { (1/quarter) }\end{array}$ & $1.9 \mathrm{E}-14$ & Footnote e & 250 \\
\hline & $\begin{array}{c}\text { gamma } \\
\text { specd } \\
\left({ }_{137} \mathrm{Cs}\right) \\
\left({ }^{134} \mathrm{Cs}\right) \\
\left({ }^{154} \mathrm{Eu}\right) \\
\left({ }^{155} \mathrm{Eu}\right) \\
\left({ }^{60} \mathrm{Co}\right)\end{array}$ & & & $\begin{array}{c}4 \\
\text { (1/quarter) }\end{array}$ & $\begin{array}{ll}1.9 & \mathrm{E}-14 \\
2.7 & \mathrm{E}-14 \\
2.3 & \mathrm{E}-14 \\
5.9 & \mathrm{E}-13 \\
1.7 & \mathrm{E}-14 \\
\end{array}$ & Footnote e & 100 \\
\hline & $239,240 \mathrm{Pu}^{\mathrm{d}}$ & & & $\begin{array}{c}4 \\
\text { (1/quarter) }\end{array}$ & $2.0 \mathrm{E}-15$ & Footnote e & 250 \\
\hline
\end{tabular}

a DOE/RL-96-68, Vol. 4, Table 7-1, states that duplicate analyses should have $<20 \%$ relative percent difference when the result is $>5$ times the minimum detectable activity.

b Performance evaluation sample/analysis results should meet the minimum acceptability scores specified by the required evaluation studies. Evaluation study requirements are specified in Section 4.0.

c MDCs shall be as low as reasonably attainable, but shall not exceed the values specified in the table. 
HNF-EP-0857-2

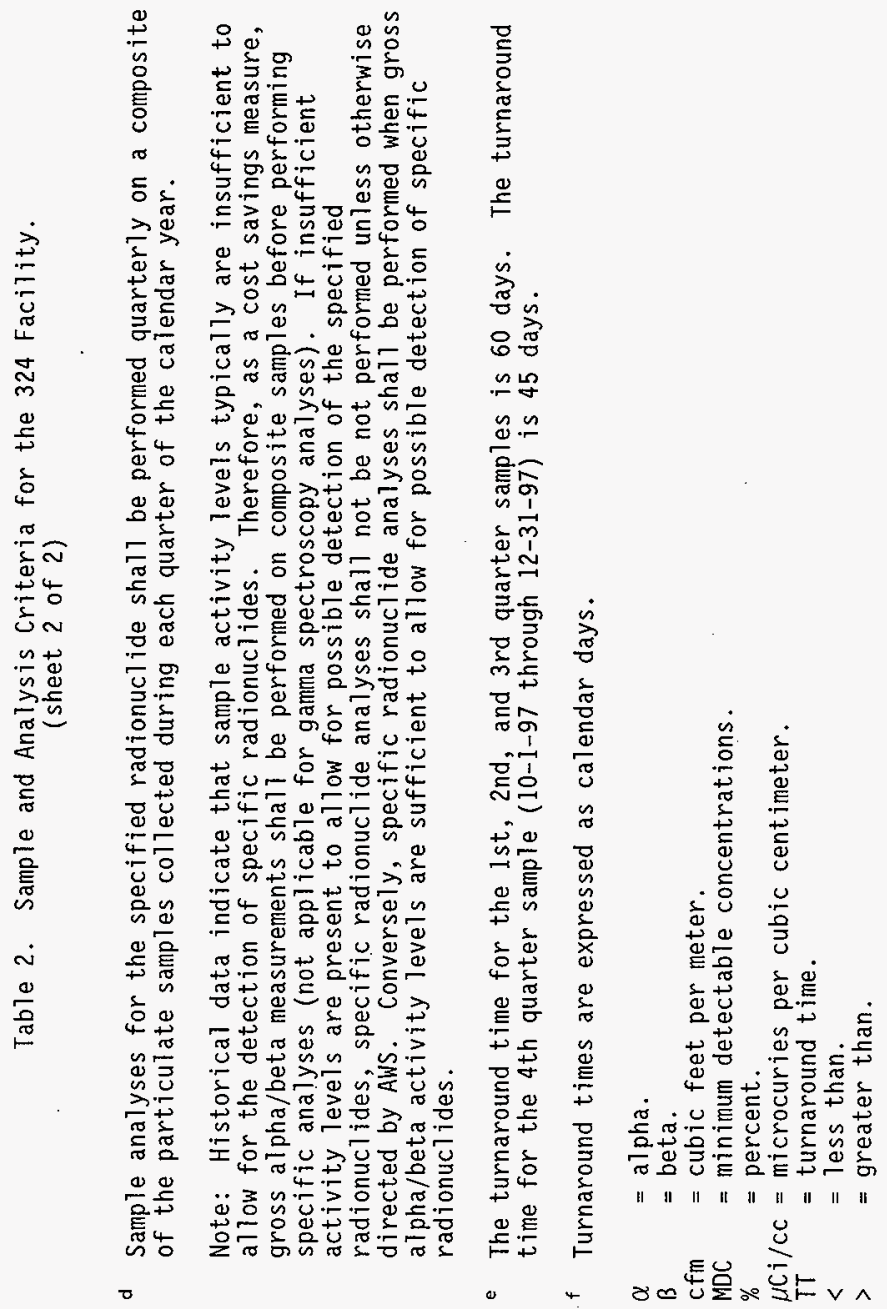


Table 3. Sample and Analysis Criteria for the 327 Facility. (sheet 1 of 3)

\begin{tabular}{|c|c|c|c|c|c|c|c|}
\hline \multicolumn{8}{|c|}{327 FACILITY, ESP-327-01-S Stack } \\
\hline \multicolumn{8}{|c|}{ SAMPLE AND ANALYSIS CRITERIA } \\
\hline Sample size & $\begin{array}{l}\text { Type of } \\
\text { analysis }\end{array}$ & Precision ${ }^{a}$ & Accuracy & $\begin{array}{l}\text { Samples per } \\
\text { year } \\
\text { (frequency) }\end{array}$ & $(\mu \mathrm{MDC} / \mathrm{cc})$ & $\begin{array}{c}\mathrm{TT}^{f} \\
(\text { days })\end{array}$ & Price, $\$$ \\
\hline \multirow{7}{*}{$\begin{array}{l}\text { nominal } 2.0 \mathrm{cfm} \\
\text { for period of } \\
\text { collection, } \\
\text { unless } \\
\text { otherwise } \\
\text { indicated }\end{array}$} & $\begin{array}{l}\text { gross } \alpha \\
\text { and } \\
\text { gross } \beta\end{array}$ & \multirow[t]{7}{*}{$<20 \%$} & \multirow[t]{7}{*}{ Footnote $b$} & $\begin{array}{c}26 \\
(1 / 2 \text { weeks })\end{array}$ & $\begin{array}{c}2.0 \mathrm{E}-15 \\
\text { and } \\
1.9 \mathrm{E}-14\end{array}$ & 14 & 100 \\
\hline & ${ }^{238} \mathrm{Pu}^{\mathrm{d}}$ & & & $\begin{array}{c}4 \\
\text { (1/quarter) }\end{array}$ & $2.1 \mathrm{E}-15$ & Footnote e & 250 \\
\hline & ${ }^{241} \mathrm{Am}^{\mathrm{d}}$ & & & $\begin{array}{c}4 \\
\text { (1/quarter) }\end{array}$ & $1.9 E-15$ & Footnote e & $250 \rightarrow$ \\
\hline & ${ }^{3} \mathrm{H}$ & & & $\stackrel{24}{(2 / \text { month })}$ & $1.5 \mathrm{E}-9$ & 45 & 750 \\
\hline & ${ }^{90} S r^{d}$ & & & $\begin{array}{c}4 \\
\text { (1/guarter) }\end{array}$ & $1.9 E-14$ & Footnote e & 250 \\
\hline & $\begin{array}{l}\text { gamma } \\
\text { spec d } \\
\left({ }^{137} \mathrm{Cs}\right) \\
\left({ }^{134} \mathrm{Cs}\right) \\
\left({ }^{154} \mathrm{Eu}\right) \\
\left({ }^{155} \mathrm{Eu}\right) \\
.\left({ }^{60} \mathrm{Co}\right)\end{array}$ & & & $\stackrel{4}{4}$ & $\begin{array}{ll}1.9 & \mathrm{E}-14 \\
2.7 & \mathrm{E}-14 \\
2.3 & \mathrm{E}-14 \\
5.9 & \mathrm{E}-13 \\
1.7 & \mathrm{E}-14 \\
\end{array}$ & Footnote e & 100 \\
\hline & $239,240 \mathrm{Pu}^{\mathrm{d}}$ & & & $\begin{array}{c}4 \\
\text { (1/quarter) }\end{array}$ & $2.0 \mathrm{E}-15$ & Footnote e & 250 \\
\hline
\end{tabular}


Table 3. Sample and Analysis Criteria for the 327 Facility. (sheet 2 of 3)

\begin{tabular}{|c|c|c|c|c|c|c|c|}
\hline \multicolumn{8}{|c|}{327 FACILITY, ESP-327-02-V Stack } \\
\hline \multicolumn{8}{|c|}{ SAMPLE AND ANALYSIS CRITERIA } \\
\hline Sample size & $\begin{array}{l}\text { Type of: } \\
\text { analysis }\end{array}$ & Precision & Accuräcy & $\begin{array}{l}\text { Samples/year } \\
\text { (frequency) }\end{array}$ & $\begin{array}{c}M D C^{c}, \\
(\mu \mathrm{C} j / \mathrm{CC}) \\
\end{array}$ & $\begin{array}{c}\text { Tो } \\
\text { (days) }\end{array}$ & $\begin{array}{l}\text { Unit } \\
\text { price, } \$\end{array}$ \\
\hline \multirow{6}{*}{$\begin{array}{l}\text { nominal } 2.0 \mathrm{cfm} \\
\text { for period of } \\
\text { collection, } \\
\text { unless } \\
\text { otherwise } \\
\text { indicated }\end{array}$} & $\begin{array}{c}\text { gross } \alpha \\
\text { and } \\
\text { gross } B\end{array}$ & \multirow[t]{6}{*}{$<20 \%$} & \multirow[t]{6}{*}{ Footnote b } & (1/2 26 weeks) & $\begin{array}{c}2.0 \mathrm{E}-15 \\
\text { and } \\
1.9 \mathrm{E}-14\end{array}$ & 14 & 100 \\
\hline & ${ }^{238} \mathrm{Pu}^{\mathrm{d}}$ & & & $\begin{array}{c}4 \\
\text { (1/quarter) }\end{array}$ & $2.1 \mathrm{E}-15$ & Footnote e & 250 \\
\hline & ${ }^{241} \mathrm{Am}^{\mathrm{d}}$ & & & $\begin{array}{c}4 \\
\text { (1/quarter) }\end{array}$ & $1.9 E-15$ & Footnote e & 250 \\
\hline & ${ }^{90} \mathrm{Sr}$ & & & $\stackrel{4}{(1 / \text { quarter })}$ & $1.9 \mathrm{E}-14$ & Footnote e & 250 \\
\hline & $\begin{array}{l}\text { gamma } \\
\text { spec } \\
\left({ }^{137} \mathrm{Cs}\right) \\
\left({ }^{134} \mathrm{Cs}\right) \\
\left({ }^{154} \mathrm{Eu}\right) \\
\left({ }^{155} \mathrm{Eu}\right) \\
\left({ }^{60} \mathrm{Co}\right)\end{array}$ & & & $\stackrel{4}{\text { (1/quarter) }}$ & $\begin{array}{ll}1.9 & \mathrm{E}-14 \\
2.7 & \mathrm{E}-14 \\
2.3 & \mathrm{E}-14 \\
5.9 & \mathrm{E}-13 \\
1.7 & \mathrm{E}-14\end{array}$ & Footnote e & 100 \\
\hline & $239,240 \mathrm{Pu}^{\mathrm{d}}$ & & & $\begin{array}{c}4 \\
\text { (1/quarter) }\end{array}$ & $2.0 \mathrm{E}-15$ & Footnote e & 250 \\
\hline
\end{tabular}


a DOE/RL-96-68, Vo1. 4, Table 7-1, states that duplicate analyses should have $<20 \%$ relative percent difference when the result is $>5$ times the minimum detectable activity.

b Performance evaluation sample/analysis results should meet the minimum acceptability scores specified by the required evaluation studies. Evaluation study requirements are specified in Section 4.0 .

c MDCs shall be as low as reasonably attainable, but shall not exceed the values specified in the table.

d Sample analyses for the specified radionuclide shall be performed quarterly on a composite of the particulate samples collected during each quarter of the calendar year.

Note: Historical data indicate that sample activity levels typically are insufficient to allow for the detection of specific radionuclides. Therefore, as a cost savings measure, gross alpha/beta measurements shall be performed on composite samples before performing specific anatyses (not applicable for gamma spectroscopy analyses). If insufficient activity levels are present to allow for possible detection of the specified

radionuclides, specific radionuclide analyses shall not be not performed unless otherwise directed by AWS. Conversely, specific radionuclide analyses shall be performed when gross alpha/beta activity levels are sufficient to allow for possible detection of specific radionuclides.

e The turnaround time for the 1st, 2nd, and 3rd quarter samples is 60 days. The turnaround time for the 4th quarter sample (10-1-97 through 12-31-97) is 45 days.

$f$ Turnaround times are expressed as calendar days.

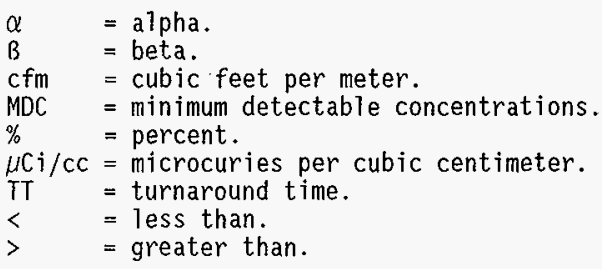


Table 4. Sample and Analysis Criteria for the 340 Facility. (sheet 1 of 2)

\begin{tabular}{|c|c|c|c|c|c|c|c|}
\hline \multicolumn{8}{|c|}{340 FACILITY, 340-NT-EX Stack } \\
\hline \multicolumn{8}{|c|}{ SAMPLE AND ANALYSIS CRITERIA } \\
\hline Sample size & $\begin{array}{l}\text { Type of } \\
\text { analysis }\end{array}$ & Precisiona & Accuracy & $\begin{array}{l}\text { Samples/year } \\
\text { (frequency) }\end{array}$ & $\begin{array}{r}M D C^{c} \\
(\mu C \mathrm{Cl} / \mathrm{CC})\end{array}$ & $\begin{array}{c}T^{f} \\
(\text { days) }\end{array}$ & $\begin{array}{l}\text { Unit } \\
\text { price, } \$\end{array}$ \\
\hline \multirow{6}{*}{$\begin{array}{l}\text { nominal } 3.1 \mathrm{cfm} \\
\text { for period of } \\
\text { collection, } \\
\text { unless } \\
\text { otherwise } \\
\text { indicated }\end{array}$} & $\begin{array}{c}\operatorname{gross} \alpha \\
\text { and } \\
\text { gross } \beta\end{array}$ & \multirow[t]{6}{*}{$<20 \%$} & \multirow[t]{6}{*}{ Footnote $b$} & (1/2 26 weeks) & $\begin{array}{c}2.0 \mathrm{E}-15 \\
\text { and } \\
1.9 \mathrm{E}-14\end{array}$ & 14 & 100 \\
\hline & ${ }^{238} \mathrm{Pu}^{\mathrm{d}}$ & & & $\begin{array}{c}4 \\
\text { (1/quarter) }\end{array}$ & $2.1 \mathrm{E}-15$ & Footnote e & 250 \\
\hline & ${ }^{241} \mathrm{Am}^{\mathrm{d}}$ & & & $\begin{array}{c}4 \\
\text { (1/quarter) }\end{array}$ & $1.9 E-15$ & Footnote e & 250 \\
\hline & ${ }^{131} \mathrm{I}$ & & & $\begin{array}{c}4 \\
\text { (1/quarter) }\end{array}$ & $2.1 \mathrm{E}-13$ & 14 & 100 \\
\hline & $\begin{array}{l}\text { gamma } \\
\text { spec }^{\text {d }} \\
\left({ }^{137} \mathrm{Cs}\right)\end{array}$ & & & $\begin{array}{c}4 \\
\text { (1/quarter) }\end{array}$ & $1.9 \mathrm{E}-14$ & Footnote e & 100 \\
\hline & ${ }^{239}, 240 \mathrm{Pu}^{\mathrm{d}}$ & & & $\begin{array}{c}4 \\
\text { (1/quarter) } \\
\end{array}$ & $2.0 \mathrm{E}-15$ & Footnote e & 250 \\
\hline \multicolumn{8}{|c|}{340 FACILITY, 340-DECON Stack } \\
\hline \multicolumn{8}{|c|}{ SAMPLE AND ANALYSIS CRITERIA } \\
\hline Sample size & $\begin{array}{l}\text { Type of } \\
\text { analysis }\end{array}$ & Precision $^{a}$ & Accuracy & $\begin{array}{l}\text { Samples/year } \\
\text { (frequency) }\end{array}$ & $\begin{array}{l}M D C \\
H C 1 / C C\end{array}$ & $\begin{array}{r}\operatorname{Tr}^{\mathrm{f}} \\
\text { (days) }\end{array}$ & $\begin{array}{l}\text { Unit } \\
\text { price, } \$\end{array}$ \\
\hline $\begin{array}{l}\text { nominal } \\
2 \text { cfm for } \\
\text { period of } \\
\text { collection, } \\
\text { unless } \\
\text { otherwise } \\
\text { indicated }\end{array}$ & $\begin{array}{c}\text { gross } \alpha \\
\text { and } \\
\text { gross } B\end{array}$ & $<20 \%$ & Footnote $b$ & $\begin{array}{c}4 \\
\text { (1/quarter) }\end{array}$ & $\begin{array}{l}2.0 \mathrm{E}-15 \\
\text { and } \\
1.9 \mathrm{E}-14\end{array}$ & 14 & 100 \\
\hline
\end{tabular}


Table 4. Sample and Analysis Criteria for the 340 Facility. (sheet 2 of 2)

\begin{tabular}{|c|c|c|c|c|c|c|c|}
\hline \multicolumn{8}{|c|}{340 FACILITY, 340-B-BLDG Stack } \\
\hline \multicolumn{8}{|c|}{ SAMPLE AND ANALYSIS CRITERIA } \\
\hline Sample Size & $\begin{array}{l}\text { Type of } \\
\text { Analysis } \\
\end{array}$ & Precision ${ }^{a}$ & Accuracy & $\begin{array}{l}\text { Samples/year } \\
\text { (frequency) }\end{array}$ & $\begin{array}{l}M \mathrm{MC}^{\mathrm{C}} \\
\mu \mathrm{C} 1 / \mathrm{CC}\end{array}$ & $\begin{array}{l}\text { TI } \\
\text { (days) }\end{array}$ & Unit \\
\hline $\begin{array}{l}\text { nominal } \\
2 \text { cfm for } \\
\text { perjod of } \\
\text { collection, } \\
\text { unless } \\
\text { otherwise } \\
\text { indicated }\end{array}$ & $\begin{array}{l}\operatorname{gross} \alpha \\
\text { and } \\
\text { gross } \beta\end{array}$ & $<20 \%$ & Footnote $b$ & $\stackrel{4}{\text { (1/quarter) }}$ & $\begin{array}{l}2.0 \mathrm{E}-15 \\
\text { and } \\
1.9 \mathrm{E}-14\end{array}$ & 14 & 100 \\
\hline
\end{tabular}

DOE/RL-96-68, Vol. 4, Table 7-1, states that duplicate analyses should have $<20 \%$ relative percent difference when the result is $>5$ times the minimum detectable activity.

b

Performance evaluation sample/analys is results should meet the minimum acceptability scores specified by the required evaluation studies. Evaluation study requirements are specified in section 4.0.

c MDCs shall be as low as reasonably attainable, but shall not exceed the values specified in the table.

d

Sample analyses for the specified radionuclide shall be performed quarterly on a composite of the particulate samples collected during each quarter of the calendar year.

Note: Historical data indicate that sample activity levels typically are insufficient to allow for the detection of specific radionuclides. Therefore, as a cost savings measure, gross alpha/beta measurements shall be performed on composite samples before performing specific analyses (not applicable for gamma spectroscopy analyses). If insufficient activity levels are present to allow for possible detection of the specified radionuclides, specific radionuclide analyses shall not be not performed unless otherwise directed by AwS. Conversely, specific radionuclide analyses shall be performed when gross alpha/beta activity levels are sufficient to allow for possible detection of specific radionuclides.

e

The turnaround time for the 1st, 2nd, and 3rd quarter samples is 60 days. The turnaround time for the 4 th quarter sample $(10-1-97$ through $12-31-97)$ is 45 days.

$f$ Turnaround times are expressed as calendar days:
$\alpha \quad=$ alpha
$<=$ less than.
$B=$ beta.
$\mathrm{cfm}=$ cubic feet per meter.
MDC = minimum detectable concentrations.
$\% \quad$ percent .
$\mu \mathrm{C} i / \mathrm{cc}=$ microcuries per cubic centimeter .
$\mathrm{TT}=$ turnaround time. 
Table 5. Sample and Analysis Criteria for the Fast Flux Test Facility. (sheet 1 of 2)

\begin{tabular}{|c|c|c|c|c|c|c|c|}
\hline \multicolumn{8}{|c|}{ FFTF, FFTF-CB-EX Stack } \\
\hline Sample size & $\begin{array}{l}\text { Type of } \\
\text { analysis }\end{array}$ & Precision & Accuracy & $\begin{array}{l}\text { Samples/year } \\
\text { (frequency) }\end{array}$ & $M \mathrm{MOC} / \mathrm{cc}$ & $\begin{array}{c}T^{e} \\
\text { (days) }\end{array}$ & $\begin{array}{l}\text { Unit } \\
\text { price } \$\end{array}$ \\
\hline \multirow{2}{*}{$\begin{array}{l}\text { nominal } 2 \mathrm{cfm} \\
\text { for period of } \\
\text { collection, } \\
\text { unless } \\
\text { otherwise } \\
\text { indicated }\end{array}$} & $\begin{array}{l}\text { gross } \alpha \text { and } \\
\text { gross } \beta\end{array}$ & \multirow[t]{2}{*}{$<20 \%$} & \multirow[t]{2}{*}{ Footnote $b$} & $\begin{array}{c}52 \\
(1 / \text { week })\end{array}$ & $\begin{array}{l}2.0 \mathrm{E}-15 \\
\text { and } \\
1.9^{\mathrm{E}-14}\end{array}$ & 14 & 100 \\
\hline & ${ }^{131} \mathrm{I}$ & & & $\begin{array}{c}26 \\
(1 / 2 \text { weeks })\end{array}$ & $2.1 E-13$ & 14 & 100 \\
\hline
\end{tabular}

\begin{tabular}{|c|c|c|c|c|c|c|c|}
\hline \multicolumn{8}{|c|}{ FFTF, FFTF-RE-SB Stack } \\
\hline \multicolumn{8}{|c|}{ SAMPLE AND ANALYSIS CRITERIA } \\
\hline Sample Size & $\begin{array}{l}\text { Type of } \\
\text { Analysis. }\end{array}$ & Precision ${ }^{a}$ & Accuracy ${ }^{b}$ & $\begin{array}{l}\text { Samples/year } \\
\text { (freguency) }\end{array}$ & $\begin{array}{l}M D C^{c} \\
M C i / C C\end{array}$ & $\begin{array}{c}\text { Toe } \\
\text { (days) }\end{array}$ & Urice, $\$$ \\
\hline \multirow{2}{*}{$\begin{array}{l}\text { nominal } 2 \mathrm{cfm} \\
\text { for period of } \\
\text { collection, } \\
\text { unless } \\
\text { otherwise } \\
\text { indicated }\end{array}$} & $\begin{array}{l}\text { gross } \alpha \text { and } \\
\text { gross } \beta\end{array}$ & \multirow[t]{2}{*}{$<20 \%$} & \multirow[t]{2}{*}{ Footnote $b$} & $\begin{array}{c}52 \\
\text { (1/week) }\end{array}$ & $\begin{array}{c}2.0 \mathrm{E}-15 \\
\text { and } \\
1.9 \mathrm{E}-14\end{array}$ & 14 & 100 \\
\hline & ${ }^{131} \mathrm{I}$ & & & $\begin{array}{c}26 \\
\text { (1/2 weeks) }\end{array}$ & $2.1 \mathrm{E}-13$ & 14 & 100 \\
\hline
\end{tabular}


Table 5. Sample and Analysis Criteria for the Fast Flux Test Facility. (sheet 2 of 2)

\begin{tabular}{|c|c|c|c|c|c|c|c|}
\hline \multicolumn{8}{|c|}{ FFTF, FFTF-HT-TR Stack } \\
\hline \multicolumn{8}{|c|}{ SAMPLE AND ANALYSIS CRITERIA } \\
\hline Sample size & $\begin{array}{l}\text { Type of } \\
\text { analys is } \\
\end{array}$ & Precision & Accuracy & $\begin{array}{l}\text { Samples/year } \\
\text { (frequency })^{d}\end{array}$ & $\begin{array}{l}M D C^{c} \\
\mu \mathrm{Ci} / \mathrm{CC}\end{array}$ & $\begin{array}{l}\text { Toe } \\
\text { (days) } \\
\end{array}$ & Unitt \\
\hline $\begin{array}{l}\text { nominal } \\
2 \mathrm{cfm} \text { for } \\
\text { period of } \\
\text { collection, } \\
\text { unless } \\
\text { otherwise } \\
\text { indicated }\end{array}$ & $\begin{array}{l}\text { gross } \alpha \text { and } \\
\text { gross } \beta\end{array}$ & $<20 \%$ & Footnote $b$ & $\begin{array}{c}52 \\
\text { (1/week) } \\
\end{array}$ & $\begin{array}{l}2.0 \mathrm{E}-15 \\
\text { and } \\
1.9 \mathrm{E}-14\end{array}$ & 14 & 100 \\
\hline
\end{tabular}

a $D 0 E / R L-96-68, V_{01}$. 4, Table 7-1, states that duplicate analyses should have $<20 \%$ relative percent difference when the result is $>5$ times the minimum detectable activity.

b Performance evaluation sample/analysis results should meet the minimum acceptability scores specified by the required evaluation studies. Evaluation study requirements are specified in Section 4.0 .

c MDCs shall be as low as reasonably attainable, but shall not exceed the values specified in the table.

d Sample frequency may be reduced during the calendar year. A reduction in the sample frequency may result in less samples delivered to PNNL for analysis.

e Turnaround times are expressed as calendar days.

$f$ Four samples will be collected during a 4 week period ( 1 sample per week). The samples shall be analyzed as a batch to reduce analytical costs per sample.

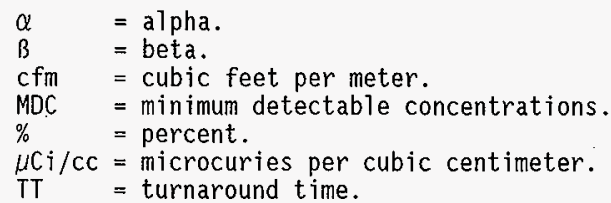


Table 6. Sample and Analysis Criteria for the 437 Maintenance and Storage Facility. (sheet 1 of 2)

\begin{tabular}{|c|c|c|c|c|c|c|c|}
\hline \multicolumn{8}{|c|}{ MASF, 437-MN\&ST Stack } \\
\hline Sample size & $\begin{array}{l}\text { Type of } \\
\text { analysis } \\
\end{array}$ & Precision ${ }^{a}$ & Accuracy ${ }^{b}$ & $\begin{array}{l}\text { Samples/year } \\
\text { (frequency) }\end{array}$ & $\begin{array}{l}M D C^{c} \\
\mu C 1 / \mathrm{CC}\end{array}$ & $\begin{array}{c}\text { TTe } \\
\text { (days) }\end{array}$ & $\begin{array}{l}\text { Unit } \\
\text { price, } \$\end{array}$ \\
\hline $\begin{array}{l}\text { nominal } \\
2.0 \mathrm{cfm} \text { for } \\
\text { period of } \\
\text { collection, } \\
\text { unless } \\
\text { otherwise } \\
\text { indicated }\end{array}$ & $\begin{array}{l}\text { gross } \alpha \text { and } \\
\text { gross } \beta\end{array}$ & $<20 \%$ & Footnote $b$ & $\begin{array}{c}52 \\
\text { (1/week) }\end{array}$ & $\begin{array}{l}2.0 \mathrm{E}-15 \\
\text { and } \\
1.9 \mathrm{E}-14\end{array}$ & 14 & 100 \\
\hline
\end{tabular}

\begin{tabular}{|c|c|c|c|c|c|c|c|}
\hline \multicolumn{8}{|c|}{ MASF, 437-1-61 Stack } \\
\hline \multicolumn{8}{|c|}{ SAMPLE AND ANALYSIS CRITERIA } \\
\hline Sample size & $\begin{array}{l}\text { Type of } \\
\text { analys is } \\
\end{array}$ & Precision ${ }^{a}$ & Accuracy & $\begin{array}{c}\text { Samples / year } \\
\text { (frequency) }\end{array}$ & $\begin{array}{l}M D C^{c} \\
\mu \mathrm{CI} / \mathrm{CC}\end{array}$ & $\begin{array}{c}\mathrm{TT}^{f} \\
\text { (days) } \\
\end{array}$ & $\begin{array}{c}\text { Unit } \\
\text { price, } \$\end{array}$ \\
\hline $\begin{array}{l}\text { nominat } \\
2 \mathrm{cfm} \text { for } \\
\text { period of } \\
\text { collection, } \\
\text { unless } \\
\text { otherwise } \\
\text { indicated }\end{array}$ & $\begin{array}{c}\text { gross } \alpha \\
\text { and } \\
\text { gross } B\end{array}$ & $<20 \%$ & Footnote b & 4 & $\begin{array}{c}2.0 \mathrm{E}-15 \\
\text { and } \\
1.9 \mathrm{E}-14\end{array}$ & 14 & 100 \\
\hline
\end{tabular}


Table 6. Sample and Analysis Criteria for the 437 Maintenance and Storage Facility. (sheet 2 of 2)

a DOE/RL-96-68, Vol. 4, Table 7-1, states that duplicate analyses should have $<20 \%$ relative percent difference when the result is $>5$ times the minimum detectable activity.

b Performance evaluation sample/analysis results should meet the minimum acceptability scores specified by the required evaluation studies. Evaluation study requirements are specified in Section 4.0 .

c MDCs shall be as low as reasonably attainable, but shall not exceed the values specified in the table.

d Sample frequency may be reduced during the calendar year. A reduction in the sample frequency may result in less samples delivered to PNNL for analysis.

e Turnaround times are expressed as calendar days.

$\alpha \quad=$ a pha.

B = beta.

cfm = cubic feet per meter.

MDC = minimum detectable concentrations.

$\% \quad=$ percent.

$\mu \mathrm{Ci} / \mathrm{cc}=$ microcuries per cubic centimeter .

TT = turnaround time.

$<\quad=$ less than.

$>\quad=$ greater than. 
HNF-EP-0857-2

Table 7. Environmental Reiease Summary Reporting Parameters. (sheet 1 of 2)

\begin{tabular}{|c|c|c|c|c|}
\hline $\begin{array}{l}\text { EDP } \\
\text { code }\end{array}$ & File title & $\begin{array}{l}\text { Stack code } \\
\text { or } \\
\text { Stream code }\end{array}$ & $\begin{array}{l}\text { Companion } \\
\text { files }\end{array}$ & $\begin{array}{l}\text { Reportable } \\
\text { radionuclides }\end{array}$ \\
\hline F002 & 340 Vault Tank Exhaust & 340-NT-EX & $\begin{array}{l}\mathrm{F} 602 \\
\mathrm{~F} 017\end{array}$ & $\begin{array}{l}\text { alpha } \\
\text { beta }\end{array}$ \\
\hline F008 & 340 B Building & $340-B-B L D$ & none & $\begin{array}{l}\text { alpha } \\
\text { beta }\end{array}$ \\
\hline F009 & $\begin{array}{l}340 \text { Decontamination } \\
\text { Facility }\end{array}$ & 340-DECON & none & $\begin{array}{l}\text { alpha } \\
\text { beta }\end{array}$ \\
\hline F011 & FFTF Combined Exhaust & FFTF-CB-E & $\begin{array}{l}\text { F018 } \\
\text { F024 } \\
\end{array}$ & $\begin{array}{c}\text { alpha } \\
\text { beta }\end{array}$ \\
\hline F012 & $\begin{array}{c}\text { FFTF Lower Reactor } \\
\text { Service Building }\end{array}$ & FFTF-RESB & F016 & $\begin{array}{l}\text { alpha } \\
\text { beta }\end{array}$ \\
\hline F013. & $\begin{array}{l}\text { FFTF Reactor Heat } \\
\text { Transport System }\end{array}$ & FFTF-HT-T & none & $\begin{array}{l}\text { a.pha } \\
\text { beta }\end{array}$ \\
\hline F014 & $\begin{array}{c}\text { MASF } \\
437-M N \& S T\end{array}$ & 437-MN\&ST & none & $\begin{array}{l}\text { alpha } \\
\text { beta }\end{array}$ \\
\hline F016 & $\begin{array}{c}\text { FFTF Lower Reactor } \\
\text { Service Building } \\
\text { I-131 }\end{array}$ & FFTF-RESB & F012 & $. I-131$ \\
\hline $\mathrm{F} 017$ & $\begin{array}{c}340 \text { Vault Tank Exhaust } \\
\text { I-131 Charcoal Cart }\end{array}$ & 340-NT-EX & $\begin{array}{l}\mathrm{F} 002 \\
\mathrm{~F} 602\end{array}$ & $1-131$ \\
\hline F018 & $\begin{array}{l}\text { FFTF Combined Exhaust } \\
\mathrm{I}-131\end{array}$ & FFTF-CB-E & $\begin{array}{l}\mathrm{F} 011 \\
\mathrm{~F} 024\end{array}$ & $I-131$ \\
\hline F019 & $\begin{array}{c}\text { MASF } \\
437-1-61 \\
\end{array}$ & $437-1-61$ & none & $\begin{array}{l}\text { aipha } \\
\text { beta }\end{array}$ \\
\hline $\mathrm{F} 602$ & $\begin{array}{c}340 \text { Vault Tank Exhaust } \\
\text { Quarterly, Composite }\end{array}$ & 340-NT-EX & $\begin{array}{l}\text { F002 } \\
\text { F017 }\end{array}$ & $\begin{array}{c}\mathrm{Cs}-137 \\
\mathrm{Pu}-239,240 \\
\mathrm{Pu}-238 \\
\mathrm{Am}-241 \\
\end{array}$ \\
\hline F024 & $\begin{array}{c}\text { FFTF Combined Exhaust } \\
\mathrm{H}-3\end{array}$ & FFTF-CB-E & $\begin{array}{l}\text { F011 } \\
\text { F018 }\end{array}$ & $\mathrm{H}-3$ \\
\hline $\mathrm{F} 025$ & $E P-324-01-S$ & $.324-01-5$ & $\mathrm{~F} 028, \mathrm{~F} 603$ & $\begin{array}{c}\text { alpha } \\
\text { beta }\end{array}$ \\
\hline F026 & $E P-327-01-S$ & $327-01-5$ & $\mathrm{~F} 029, \mathrm{~F} 604$ & $\begin{array}{c}\text { alpha } \\
\text { beta }\end{array}$ \\
\hline F027 & $E P-327-02-V$ & $327-02-V$ & $\mathrm{~F} 030, \mathrm{~F} 605$ & $\begin{array}{l}\text { a)pha } \\
\text { beta }\end{array}$ \\
\hline
\end{tabular}


HNF-EP-0857-2

Table 7. Environmental Release Summary Reporting Parameters. (sheet 2 of 2)

\begin{tabular}{|c|c|c|c|c|}
\hline $\begin{array}{l}\text { EDP } \\
\text { code }\end{array}$ & File title & $\begin{array}{c}\text { Stack code } \\
\text { or. } \\
\text { Stream code }\end{array}$ & $\begin{array}{l}\text { Companion } \\
\text { files }\end{array}$ & $\begin{array}{l}\text { Reportable } \\
\text { radionuclides }\end{array}$ \\
\hline F603 & $\begin{array}{l}\text { EP-324-01-S } \\
\text { Quarterly Composite }\end{array}$ & $324-01-S$ & $\mathrm{~F} 025, \mathrm{~F} 028$ & $\begin{array}{c}\text { Pu-238 } \\
\text { Am-241 } \\
\text { Sr-90 } \\
\text { Pu-239,240 } \\
\text { Cs-137 } \\
\text { Cs-134 } \\
\text { Eu-154 } \\
\text { Eu-155 } \\
\text { Co-60 } \\
\end{array}$ \\
\hline F604 & $\begin{array}{l}\text { EP-327-01-S } \\
\text { Quarterly Composite }\end{array}$ & $327-01-5$ & F026, F029 & $\begin{array}{c}\mathrm{Pu}-238 \\
\mathrm{Am}-241 \\
\mathrm{Sr}-90 \\
\mathrm{Pu}-239,240 \\
\mathrm{Cs}-137 \\
\mathrm{Cs}-134 \\
\mathrm{Eu}-154 \\
\mathrm{Eu}-155 \\
\mathrm{Co}-60\end{array}$ \\
\hline F605 & $\begin{array}{l}\text { EP-327-02-V } \\
\text { Quarterly Composite }\end{array}$ & $327-02-V$ & $\mathrm{~F} 027, \mathrm{~F} 030$ & $\begin{array}{c}\mathrm{Pu}-238 \\
\mathrm{Am}-241 \\
\mathrm{Sr}-90 \\
\mathrm{Pu}-239,240 \\
\mathrm{Cs}-137 \\
\mathrm{Cs}-134 \\
\mathrm{Eu}-154 \\
\mathrm{Eu}-155 \\
\mathrm{Co}-60 \\
\end{array}$ \\
\hline F028 & $\begin{array}{c}E P-324-01-S \\
\mathrm{H}-3 \\
\end{array}$ & $324-01-S$ & $F 603, F 025$ & $\mathrm{H}-3$ \\
\hline F029 & $\begin{array}{c}E P-327-01-S \\
\mathrm{H}-3\end{array}$ & $327-01-5$ & $\mathrm{~F} 604, \mathrm{~F} 026$ & $\mathrm{H}-3$ \\
\hline F030 & 309-PRTR & 309-PRTR & NA & $\begin{array}{c}\text { alpha } \\
\text { beta }\end{array}$ \\
\hline
\end{tabular}

$E D P=$ electronic data processing.

FFTF = Fast Flux Test Facility.

MASF = Maintenance and Storage Facility .

MN\&ST = Maintenance and Storage.

NA $=$ Not applicable.

PRTR = Plutonium Recycle Test Reactor. 


\section{DISTRIBUT ION}

Mr. A. W. Conklin, Head

Air Emissions and Defense Waste Section Division of Radiation Protection

State of Washington Department of Health P. 0. Box 47827

01ympia, Washington 98504-7827

Fluor Daniel Hanford, Inc.

\section{S. M. Price}

Rust Federal Services of Hanford Inc.

$\begin{array}{ll}\text { R. B. Barmettlor } & \mathrm{L} 6-04 \\ \text { L. D. Berneski } & \mathrm{L} 6-04 \\ \text { W. R. Thackaberry } & \mathrm{H6}-32 \\ \text { W. E. Davis } & \mathrm{H} 6-20 \\ \text { R. W. Szelmeczka } & \mathrm{L} 6-05 \\ \text { L. P. Diediker } & \mathrm{S} 3-95 \\ \text { B. P. Gleckler } & \mathrm{S} 3-95 \\ \text { E. M. Greager } & \mathrm{H6-20} \\ \text { D. L. Halgren } & \mathrm{L} 6-04 \\ \text { J. J. Luke } & \mathrm{H6}-25 \\ \text { J. K. Perry } & \mathrm{H6-20}\end{array}$

B\&W Hanford Company

E. J. Bitten

B. C. Cornwell

N. R. Dah1

T. A. Dillhoff

D. R. Ekstrom

W. A. Hoober

D. L. Johnson

G. J. Lebaron

M. S. Wright

D. E. Rasmussen

J. M. Steffen

Lockheed Martin Services, Inc.

J. N. Diven

H8-20

Central Files

A3-88

DPC

A3-88

Pacific Northwest National Laboratory

T. L. Almeida.

P7-25

0 . P. Bredt

P7 -27

L. R. Greenwood

P7-22

K. J. Kuh T-K 1 inger

P7-27

J. M. Latkovich

P7 -27

R. G. Swoboda

P7-22

K. K. Thomas

P7-22 
HNF-EP-0857-2

\section{DISTRIBUTION}

Mr. A. W. ConkTin, Head

Air Emissions and Defense Waste Section

Division of Radiation Protection

State of Washington Department of Health

P. 0. Box 47827

Olympia, Washington $98504-7827$

Fluor Daniel Hanford, Inc.

S. M. Price

Rust Federal Services of Hanford Inc.

R. B. Barmettior

L6-04

L. D. Berneski

L6-04

W. R. Thackaberry

H6-32

W. E. Davis

R. W. Szelmeczka

H6-20

L. P. Diediker

L6-05

B. P. Gleckler

S3-95

E. M. Greager

S3-95

D. L. Halgren

$\mathrm{H} 6-2 \mathrm{O}$

J. J. Luke

J. K. Perry

L6-04

H6- 25

H6-2O

B\&W Hanford Company
E. J. Bitten
B. C. Cornwell
N. R. Dahi
T. A. Dillhoff
D. R. Ekstrom
W. A. Hoober
D. L. Johnson
G. J. Lebaron
M. S. Wright
D. E. Rasmussen
N1-41
L5-70
N2-57
N2-57
L6-52
L1-03
L1-05
S6-15
L1-02
J. M. Steffen
N1-47
N1-47

Lockheed Martin Services. Inc.

J. N. Diven

H8-20

Central Files

A3-88

DPC

A3-88

Pacific Northwest National Laboratory

T. L. Almeida

P7-25

O. P. Bredt

P7 -27

L. R. Greenwood

P7 -22

K. J. Kuh $7-K l$ inger

P7 -27

J. M. Latkovich

P7-27

R. G. Swoboda

P7-22

K. K. Thomas

-P7-22 